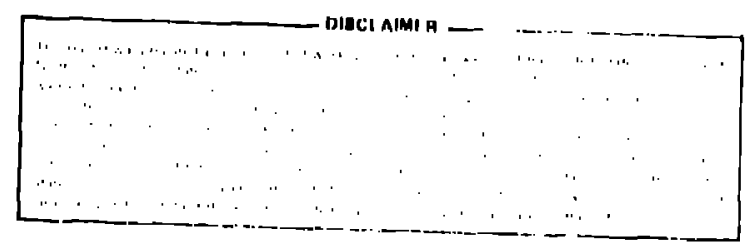




\title{
ASYMPTOTIC FLAME THEORY WITH COMPLEX CHEMISTRY
}

\section{by}

Paul C. Fife ${ }^{\dagger}$ and Basil Nicolaenko

Center for Nonlinear Studies, MS B258

Los Alamos National Laboratory

Los Alamos, NM 87545

\begin{abstract}
We investigate the structure of laminar flames with general complex chemistry networks in the Jimit of high activation energy asymptotics. Depending on the specific reaction network and other given thermomechanical data, a wide variety of flame configurations are possible. Here we present a first versinn of a systematic asymptotic reduction of complex chemistry networks and give practical criteria to determine the dominant reactions when transport and chemistry are coupled.
\end{abstract}

† Department of Mathemalics, Undversity of Arizona, Tucson, AZ 87521.

This research was partlally supported by NSF Grant 79-04443 and by the Center for Nonilinear Studies, Los Alamos National laboratory. Work also performed under the ausplces of the U.S. Department af Energy uniler cuntract W-7405-ENG-36 and contract KC-04-02-01, Division of Busic and Fingineering Sciences.

To appear, Proceedings of Specind Summer Conference on Partial Differential Equations, Durham, NII, June 1982; Americon Mathematical Society. 


\section{INTRODUCTION}

Mathematically, the subject of this presentation can be considered to be traveling waves for reaction-diffusion systems

$$
\mathrm{u}_{\mathrm{t}}=\mathrm{DU} \mathrm{zz}_{\mathrm{z}}+\mathrm{f}(\mathrm{U})
$$

where $U \in R^{n}, D$ is a positive definite transport matrix, and $f$ has the form

$$
f(U)=\sum_{j=1}^{m} w_{j}(U) K_{j}
$$

Here $w_{j}$ are scalar functions, $K_{j} \in k^{n}$, and each $w_{j}$ depends in a certain way on a small parameter $\varepsilon_{j}$. Typically,

$$
w_{j}(U)=k_{j}\left(\varepsilon_{j}\right) \psi_{j}\left(\frac{U-U}{\varepsilon_{j}}\right)
$$

for certain reference vectors $U_{j}$, constants $k_{j}$, and nonnegative functions $\psi_{j}$ with $\psi_{j}(0)=0$.

Physically, the traveling wave represents a combustion front in a premixed reactive gas. Then the components of $I$ sperify the dimensionless Lemperature and the concentrations of the reuctants. There nre m chemical reactions involved in the burnting process; $w_{j}$ are their rates. The smallness of "J expresses the act that thene rates depend atrongly on temperature (the reaclions have high hetlvation enrrgy). Nearly all helunl flames have a Jarge reaction network, mont of it unkunwn. Very often ll in ...deled by network runsinting of a few renctions with high aclfuation

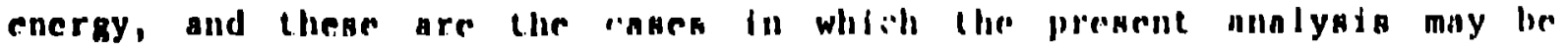
uneful. Even more commonly, only some of lhe model reactions hiuge high 
activation energy; better asymptotic procedures need to be developed for that case.

Traveling waves with velocity c are solutions of the form $U(z, t)=U(z+c t)$; but the traveling wave equation

$$
D U^{\prime \prime}-\bar{M} U^{\prime}+f(U)=0
$$

will here be written with the symbol $\bar{M}$ replacing c. llere $\bar{M}$ is mass $f l u x$, which equals velocity if the density is unity. When the density is not taken to be constant, but the pressure is (often a good approximation), the evolution equation is more complicated than (1.1); nevertheless, the problem of determining traveling wave solutions can be reduced to (1.2). We are concerned with exploiting the smallness of $\varepsilon_{j}$ to obtain approximate wave front solutions. The procedures are inked to the properties of the underlying reartion network, which supplies the vectorn $\mathrm{K}_{\mathrm{j}}$. These procedures are well known in the case whon the nelwork is modeled by a single reaclion $A \rightarrow P$. (By way of introduction, this case is reviewed in Sec. 2.) An approach similar to the one in this paper was used in treating two-reaction networks of parallel, sequential, and conpetirgfued types in $|3|$ and $|4|$, ond it was clear that the extension of this approach to more complex achemes could uncover a hiffitng nriay of posalbilteies. A Bystematic Tormaliam was called for, and hopefully is provided in pare hy like preaent paper.

The methoda are those of asymplotic analyeje. Koughly apraking, (o) lowest order thin mentus developlag rational procedusen for replactug the oilgiunl problem with a limit prohlem in which the if do not npprar expliclely. The limit problem in expected to vield an approximate 
8olution, and is easy to solve. A rigorous justification of this expectation has been $g$. $n$ recently for the case of a single reaction [1]. Many other parameters hesides $\varepsilon_{i}$ enter the problem, for example, the elements of the matrix $D$ and if the unburned state vector of the gas, $U_{-}$. Throughout the paper, we assume that these magnitudes do not interfere with the asymptotics. Particularly, we assume that any constants not depending explicitly on $\varepsilon_{i}, B_{i}$, or $\theta_{i}$ are $O(1)$ quantities. This especially refers to elements of $D$ and $D^{-1}$, reference dimensionless $T_{j}$, their differences $T_{i}-T_{j}$ and the hea: releases $Q_{j}$. These restrictions are relaxed, and a more meticulous account of the other parameters is taken in [4].

For background and previous work on asymptotic methods in flame theory, the reader is referred to [2]. A number of papers hove been written on the multiple reaction case; see the references in [3].

\section{THE CASE OF A SINGLE-REACTION MECHANISM}

Although this argument has appeared in the literature many times before, it will be convenient to review here the asymptotic anplysis of simple flames, that is, those with a single reaction $A \rightarrow P$, hence a sing]! K:

$$
D U^{\prime \prime}-\bar{M} U^{\prime}=-w(U) K, U(-\infty)=U_{-}
$$

Here $U=(T, Y)$, Lle temperature and molar concentritioll of $A, K=(Q,-I)$, nnd we lake

$$
w(U)=B Y e^{-0 / T}
$$


for positive constants $B, \theta$. As $z \rightarrow \infty$, the reaction must go to equilibrium, that is,

$$
Y(\infty)=0
$$

Integrating (2.1), we find $w(U(z))$

$$
\left[D U^{\prime}-M U\right]_{-\infty}^{\infty}=-\int_{-\infty}^{+\infty} \omega(U(z) d z K \equiv-\beta K,
$$

herce $U(\infty)-U(-\infty)=\alpha K, \alpha=\beta / M$. Applying (2.3), we find $\alpha=Y_{-}$, so

$$
U(\infty) \equiv U_{+}=U_{-}+Y_{-} K \equiv\left(T_{+}, 0\right)
$$

We define $\varepsilon=T_{+}^{2} / \theta$, and assume

$$
0<\varepsilon \ll 1
$$

In a neighborhocd of $T=T_{+}, Y=0$, we approximate

$$
\left.\omega(U)=\eta_{\varepsilon}^{\eta}\left(\frac{Y}{\varepsilon_{0}} \exp \mid\left(T-T_{+}\right) / \varepsilon\right]\right) \equiv \underset{\varepsilon}{\eta} \text { y } e^{t},
$$

where $v=(t, y) \equiv\left(U-U_{+}\right) / \therefore$, and

$$
\eta=B \varepsilon^{2} \exp \left(-\theta / \tau_{+}^{\prime}\right)
$$

This approximation is ohtained by taking the first two terms in the Taylor serins expansion of the exponent $\theta / T$ in $(2.2)$ sbout $T=T_{+}$. The remainder is $\theta(\varepsilon)$ and is discardeci. A careful analysis of this approximation is Riven in 141 . 
We next introduce new variables

$$
x=2 \eta^{\frac{1}{2}}, \bar{M}=M \eta^{-\frac{1}{2}}
$$

in (2.1) to obtain (differentiation is now with respect to $x$ )

$$
\ddot{D U}-M \dot{U}=-\frac{1}{\varepsilon} \psi\left(\frac{U-U_{+}}{\varepsilon}\right) K,
$$

where (recall $\left.u=\left(U-U_{+}\right) / \varepsilon\right)$

$$
\psi(u)=y e^{t}
$$

We may position the origin in space at will; we choose to place it where $Y=\varepsilon(y=1)$. Rewriting (2.7) with stretched variables $\xi=x / \varepsilon$, $u=\left(U-U_{+} / \varepsilon\right.$, and discarding terms formally of order $\varepsilon$, we obtain the "flame layer" equation

$$
D \frac{\partial^{2} u}{\partial \xi^{2}}+\psi(u) K=0
$$

This is to be solvef under the boundary conditions $u(\infty)=0, y(0)=1$. To do so, we observe that (2.9) and the condition $u(\infty)=0 \div \mathrm{m}, \mathrm{ly}$

$$
u=\sigma(\xi) D^{-1} K
$$

for some scalar function $\sigma$. Substituting into $(2 . y)$, we hive.

$$
\sigma^{\prime \prime}+\psi\left(\sigma D^{-1} K\right)=0, \sigma(\omega)=0 .
$$

This hos a first integral

$$
\left(\sigma^{\prime}\right)^{2}-\Omega(\sigma)=0 .
$$


where $\Omega(\sigma)=2 \int_{\sigma}^{0} \psi\left(s D^{-1} K\right)$ ds. Assuming that $D^{-1} K$ has first component positive and the second negative, we see from (2.8) that $\Omega(-\infty)$ exists. Thus the layer problem has a unique sulution, and

$$
D \frac{\partial u}{\partial \xi}(-\infty)=\beta \hat{k},
$$

where $\beta=\left(\Omega(-\infty)^{\frac{1}{2}}\right.$. Matching with the solution outside the flame layer requires, in the limit $\varepsilon \rightarrow 0$, that

$$
\operatorname{DU}(0-)=\beta K, \operatorname{DU}(0+)=0 \text {. }
$$

F.nally, for $x<0, T<T+$ and the strong dependence of $\psi$ on $T$ shows that it can be neglected in (2.7). For $x>0, Y$ is already nearly zero if $\varepsilon$ is small, so $\psi$ can again be neglected. What is left is the problem

$$
\begin{aligned}
& \ddot{D U}-\dot{M U}=0, x \neq 0, \\
& {[D \dot{U}]_{0-}^{0+}=-\beta \mathrm{K},} \\
& U( \pm \infty)=U \pm .
\end{aligned}
$$

This problem has a unique solution. Integration of (2.11) reveals (from (2.4)),

$$
[D \dot{U}]_{-\infty}^{0-}=M[U]_{-\infty}^{0-}=M\left(U_{+}-V_{-}\right)=M K_{-}^{K}
$$

Adding this to (2.12) above

$$
0=[D U]_{-\infty}^{0+}=M Y_{-} K-\beta K .
$$


Hence,

$$
M=\beta / Y_{-} .
$$

And with $M$ known, (2.11) can be integrated backward with initial conditions at $x=0$ to yield the total profile for $x<0$. For $x>0$, the only solution is $\mathrm{U} \equiv \mathrm{U}_{+}$.

In summary, asymptotics have shown the way to a limit problem (2.11) - (2.13) with nonlinearity replaced by a jump condition 2.12$)$. The coefficient $\beta$ is known independently from a layer analysis, and $(M, U(x))$ are to be determined. In the original variables, $U$ and $\bar{M}$ art determined by reversing the transformation $(2.6)$.

\section{FEASIBLE ALLOCATIONS}

We hegin by assuming given a reaction network, which is simply a specification of which reactions take place in the combustion process. It is usually sympbolized by arrows, such as:

$$
A_{1}+A_{2} \rightarrow A_{3}, 2 A_{2}+A_{3} \rightarrow P \quad, A_{1} \rightarrow 2 A
$$

(In thi:: example, there are three reactions involving three reacting

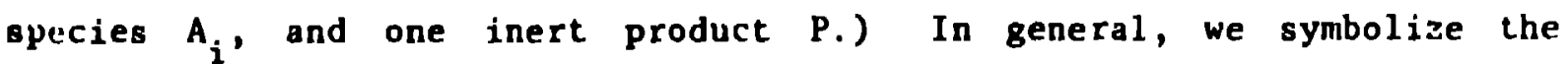
reactions by $R_{i}$, the total numter of reactions by $m$, and the number of species by $n$.

The vector $Y=\left(Y_{1}, \ldots, Y_{n}\right)$ signifies mass concentration of the various species, and will be a function of space and time. We will be 
concerned only with nonnegative concentrations, so restrict $Y$ to the nonnegative cone $\mathcal{Y}$ of $n$-dimensional (species) space.

Associated with each reaction $R_{j}$ is a reaction vector $V_{j} \in \mathbb{F}$ It represencs the net change in the number of units of the various species during a reaction event of reaction $R_{j}$. For example, in the above network (3.1), the three reaction vectors are $(-1,-1,1),(0,-2,-1)$, and $(1,0,0)$. Our basic assumption about these vectors is as follows:

Assumption 1. If $\sum_{j=1}^{m} \alpha_{j} v_{j}=0$ and $\alpha_{j} \geq 0$, then $\alpha_{j} \equiv 0$.

This assumption excludes, for example, reversible reactions, in which $v_{2}=-v_{1}$ (say). More generally, it assures, as we shall see, that equilibrium can only be reached when each reaction rate is zero.

Each reaction $R_{j}$ is assumed to have a reaction rate $w_{j} \geq 0$ which is a function of $\mathrm{Y}$ and of the temperature $\mathrm{T}$.

Def: $Y \in Y$ is an equilibrium state if

$$
\sum_{j=1}^{m} w_{j}(Y, T) v_{j}=0
$$

It follows from (3.2) that this can only happen if each $w_{j}=0$.

For each $i=1, \ldots, m$, we denote by $J(i)$ the set of all indices $j$ of species $A_{j}$ entering reaction $R_{i}$ (appearing on the left side of the arrow). Eventually, a specific form will be assumed for the $u_{i}$; but at this point we assume $w_{i}=0$ if and only if $Y_{j}=0$ for some $j \in . T(i)$. We symbolize the equilihrium cone for each reaction, and the total equilibrium cone, as 
follows:

$$
\begin{aligned}
& \xi_{i}=\left\{Y \in \mathscr{Y}: Y_{j}=0 \text { for some } j \in J(i)\right\}, \\
& \xi=\bigcap_{i=1}^{m} \xi_{i} .
\end{aligned}
$$

Def: Given an input state $Y_{-} e \mathscr{Y}_{1}$ an allocation of $Y_{\text {_ }}$ to the network is a set of numbers $\alpha_{i} \geq 0, i=1, \ldots, m$, such that

$$
Y_{-}+\sum_{i=1}^{m} \alpha_{i} v_{i} \text { e } \xi
$$

Ex. 1. In the network (3.1), we have $\xi=\left\{Y=\left(Y_{1}, Y_{2}, Y_{3}\right) \geq 0: Y_{1}=0\right.$, and $Y_{2}$ or $\left.Y_{3}=0\right\}$. Let $Y_{-}=(1,1,1)$. Then the only allocacion is $\alpha_{1}=1, \alpha_{2}=\frac{1}{2}, \alpha_{3}=0$.

Ex. 2: $\quad A_{1} \stackrel{R_{1}}{\rightarrow} 2 A_{2}+P_{1}, A_{2} \stackrel{R_{2}}{\rightarrow} 2 A_{2}+P_{2}, Y_{-}=(1,1)$. Here $\xi=\{0\}, V_{1}=(-1,2)$, $V_{2}=(2,-1)$. The reader may check that (3.2) is satisfied, but that no allocation exists. Thus, the existence of an allocation is not always guaranteed.

Ex. 3:

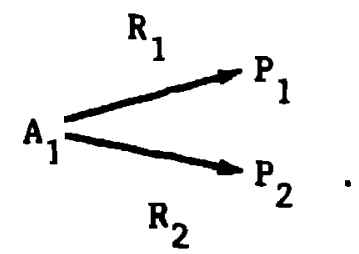

Here $\xi=\{0\}, Y_{-}$is a scalar, and $\left(\alpha_{1}, \alpha_{2}\right)$ is an allocation iff $\alpha_{1}+\alpha_{2}=$ $Y_{-}, a_{1} \geq 0$. So, of course, allocations are not necessarily unique. 
Besides concentrations, we are also concerned with temperature, so us: $n+1$-component state vectors $U=\left(T, Y_{1}, \ldots, Y_{n}\right)=(T, Y)$. Associated with each reaction is a heat release $Q_{i}>0$. We define the $(n+1)$ component vectors

$$
K_{i}=\left(Q_{i}, v_{i}\right),
$$

and shall. refer to $Q_{i}$ as the oth component of $k_{i}$.

Our basic goal, now, is to find approximations to bounded solutions $(U(x), M)$ of the problem

$$
\ddot{D U}-M \dot{U}=w_{j=1}^{m} w_{j}\left(U, c_{j}\right) K_{j}, U(-\infty)-U_{-}=\left(T_{-}, Y_{-}\right)
$$

Here we have indicated the dependence of the rates $w_{j}$ on large posjtive parameters $e_{j}$ (activalion temperatures). Specifically, we tare

$$
w_{i}=B_{i} \prod_{j \in J(i)} Y_{j}^{v(j, i)} e^{-\theta_{i} / T}
$$

where $B_{i}>0$ also might be large or small, and $v(j, i)$ is the stoichiometric coefficient of species $j$ on the lett side of reaction $i$.

The procedure will be to constïrt a suitable reduced problen in which each nonlinear term $w_{j} K_{j}$ is replaced by a jump condition in DUं at some point $x_{j}$, not known in advance. The reduced problem will be fashioned in terms of "feasible allocarions," which we pruceed to define.

Suppose an allocation $a^{\prime}=\left(\alpha_{1}, \ldots, \alpha_{m}\right)$ is given. Let

$$
U_{+}=U_{-}+{ }_{j=1}^{m} \alpha_{j} k_{j} .
$$


In particular,

$$
\begin{aligned}
& T_{+}=T_{+}(\alpha)=T_{-}+\sum_{j=1}^{m} \alpha_{j} Q_{j} \\
& \text { Using tre constants in }(3.7) \text {, we define } m \text { functions } \\
& H_{j}(T)=\varepsilon\left(\ln B_{j}-\theta_{j} / T\right),
\end{aligned}
$$

where

$$
\varepsilon \equiv \min _{j}\left(T_{+}^{2} / \theta_{j}\right)
$$

We define temperatures $T_{j}, j=1, \ldots, m$, such that

$H_{j}\left(T_{j}\right)$ is independent of $j$, and

$\underset{j}{\operatorname{Max}} T_{j}=T_{+}$

Prop. 3.1. There exists a unique temperature sequenc, $\left\{T_{j}\right\}$ satisfying (3.12).

Proof. Choose the index $k$ so that $H_{k}\left(T_{+}\right)=\underset{j}{\operatorname{Min} H_{j}}\left(T_{+}\right)$. We set $T_{k}=T_{+}$, and define the $T_{j}$ by $H_{j}\left(T_{j}\right)=H_{h}\left(T_{+}\right)$. Since $u 11 H_{j}$ are increasing functions of $T$, it follows that all $T_{j} \leq T_{+}$, so there exists such a sequence. Thxs argument also shows there is only one such sequence.

We now reorder the reaclions in order of increasing $T_{j}$, so that $\mathrm{T}_{1} \leq \mathrm{T}_{2} \leq \cdots \leq \mathrm{T}_{\mathrm{n}}=\mathrm{T}_{+}$. It can happen that some of the $\mathrm{T}_{\mathrm{j}}$ coincide. In fact, Eximple 2 ac the end of this section indicates that to be "feasible," 
an allocation may well have to be adjusted so thai two of them do coincide. (However, il seems unlikely that the case when three are the same is important.) Let $\left\{\mathrm{T}_{i}^{*}\right\}, i=1, \ldots, \mathrm{m}^{*} \leq \mathrm{m}$ be the (possibly shorter) sequence of distinct temperatures among the $T_{j}$; we denote the aumber of them by $\mathbf{m}^{*}$.

It also may happen that two of the $T_{j}$ almost coincide, that is, $0<\left|T_{i}-T_{j}\right|=O\left(\varepsilon_{i}\right)$. This case is considered non generic, and is hereby excluded from consideration. Thus, we assume that two temperatis es $T_{i}, T_{j}$ either coincide (this may be required by the feasibility criterion given later) or differ by a quantitiy of larger order than $\varepsilon_{i}+\varepsilon_{j}$.

Our attempt will be to construct a combustion front in which, roughly speaking, $m^{*}$ sets of reactions go to completion at the temperalure $T_{j}^{*}$ in that same order as one pruceeds arross the front's profile in the (upstream) direction of increasing temperatures. For that purpose, we use the notion of suballocation.

Def: For each $\ell \leq m^{*}$, suballocations a ${ }^{(l)}$ are defined to be allocations for the subnetwork consisting only of the $R_{j}$ fol $T_{j} \leq T_{\ell}^{\star}$. Let $k_{\ell}$ be the number of such reactions.

Thus, $\alpha^{(\ell)}=\left(\alpha_{1}^{(l)}, \ldots, \alpha_{k_{l}}^{(l)}\right)$, but for comparison purposes, we extend them to be sequences of length $m$ by defining $\alpha_{j}^{(\ell)}=0$ for $j>k_{\ell}$. We say $\alpha^{(l)} \leq \alpha^{\left(l^{\prime}\right)}$ if $\alpha_{j}^{(l)} \leq \alpha_{j}^{\left(\ell^{\prime}\right)}$ for all $j$. 
The situation we envisage is when there exist suballocations $\alpha^{(\ell)}$ for all $2 \leq \mathbf{m}^{*}$ satisfying

$a^{(\ell)} \leq \alpha^{(\ell+1)}, \ell=1, \ldots,{m^{*}-1}^{*} \alpha^{\left(m^{*}\right)}=\alpha$.

If such suballocations exist, we define a new set of reaction vectors v' as follows:

For each $\mathrm{k} \leq \mathbf{m}^{*}$,

$$
\alpha_{k}^{\prime} \equiv \sum_{j}\left(\alpha_{j}^{(k)}-\alpha_{j}^{(k-1)}\right)
$$

(of course taking $\left.\alpha_{j}^{(0)}=0\right)$. If $\alpha_{k}^{\prime} \neq 0$, then

$$
v_{k}^{\prime} \equiv \frac{\sum\left(\alpha_{j}^{(k)}-\alpha_{j}^{(k-1)}\right) v_{j}}{\alpha_{k}^{\prime}}
$$

If $\alpha_{k}^{\prime}=0$ for any values of $k$, we delete thuse temperatures $T_{k}^{*}$ from the sequence and accordingly renumber the temperatures, as well as the $v_{j}$, so there will be a fewer number of each. For convenience we also call the new number $\mathrm{n}^{\text {th }}$.

In sumary, we have constructed an alternative set of rectors $v_{j}$, and number $\alpha_{j}^{\prime}$, with the properties that for all $L \leq m$,

$$
Y_{-}+\sum_{j=1}^{\ell} \alpha_{j}^{\prime} v_{j}=y_{-}+\sum_{j=1}^{k_{l}} \alpha_{j}^{(l)} v_{j} \in \sum_{j=1}^{k_{l}} \xi_{j},
$$


and

$$
\sum_{j=1}^{m^{*}} \alpha_{j}^{\prime} v_{j}^{\prime}=\sum_{j=1}^{m} \alpha_{j} v_{j},
$$

on that

$$
Y_{-}+\sum_{j=1}^{m} \alpha_{j}^{\prime} v_{j}^{:}=Y_{+}(\alpha) \in \xi
$$

In some cases it may be enlightening to think of the $V_{j}^{\prime}$ as representing the reaction vector of an alternate reaction $R_{j}^{\prime}$ which is the same linear combination of the $R_{i}, i \leq j$, as is $v_{j}$ of the $v_{i}$. For instance, $j_{\text {a }}$ Case 2 of Example 3 below, $R_{2}^{\prime}$ and $R_{3}^{\prime}$ would be $A_{2} \rightarrow P$ and $1 / 3 \Lambda_{1} \rightarrow P$, respectively.

Extended vectors $K_{k}^{\prime}$ are defined to be the same linear cumbinations of the $k_{j}$ as the $v_{k}$ are of the $v_{j}(3.14)$. In particular, the first component $Q_{k}^{\prime}$ will be a linear combination of the $Q_{j}^{\prime \prime s . ~ W e ~ c a n ~ n o w ~ d e t e r m i n e ~ t h e ~}$ temperature at which the suballocation $\alpha^{(l)}$ would bring the corresponding subnetwork to equilibrium, if no reaction outside that subnetwork were present. We call it

$$
\hat{\mathrm{T}}_{\ell}=\sum_{j=1}^{\ell} \alpha_{j}^{\prime} Q_{j}^{\prime}+\mathrm{T}_{-} .
$$

Def: The allocation a is feasible if

(1) there exist, for each $\ell \leq m^{*}$, suballocations $a^{(\ell)}$ which satisfy (3.13), and

(2) $T_{f}^{*}>\hat{T}_{f}$ for $j<m^{*}$. 
Example 1. $\quad R_{1}: A_{1} \rightarrow A_{2}, \quad V_{1}=(-1,1)$

$$
\begin{aligned}
& R_{2}: A_{1}+A_{2} \rightarrow P_{1}, \quad V_{2}=(-1,-1) \\
& R_{3}: A_{2} \rightarrow P_{2}, \quad V_{3}=(0,-1) \\
& R_{4}: A_{1} \rightarrow P_{3}, \quad V_{4}=(-1,0) .
\end{aligned}
$$

These $V_{j}$ satisfy (3.2). $\xi=\{0\}$. We take $Y=(1,1)$; then all possible allocations are of the form $\alpha=(\theta, \phi, 1+\theta-\phi, 1-\theta-\phi)$, where $\theta, \phi \geq 0$ and $\theta+\phi \leq 1$. Suppose that for each $\alpha$, the new ordering is the same as the old: $T_{1} \leq T_{2} \leq T_{3} \leq T_{4}$. Then the only allocation with suballocations satisfying (3.13) is the one with $\theta=1, \phi=0$; $\alpha=(1,0,2,0)$. The sub.allocations are $\alpha^{(1)}=(i), \alpha^{(2)}=(1,0)$, $\alpha^{(3)}=(1,0,2)$, $\alpha^{(4)}=\alpha$. We then delete $R_{2}$ and $R_{4}$, so $m^{*}=2$ and the new $\alpha=(1,2)$. Cricerion (2) for feasibility still needs to be checked. If it is verified, we can expect a combustiun front to exist in which only $R_{1}$ and $R_{3}$ occur, at two different temperatures. Since there is at most one feasible allocation, we expect there is no other such cumbustion front.

Example 2. $\quad R_{1}: A \rightarrow P_{1} \quad V_{1}=-1$

$$
\begin{array}{ll}
R_{2}: A_{0} \rightarrow P_{2} & V_{2}=-1 \\
R_{3}: A+P_{3} & V_{3}=-1 .
\end{array}
$$

Again, $\xi=\{0\}$. For $Y_{-}=1$, $\| 11$ possible allocations are $\alpha=\left(\alpha_{1}, \alpha_{2}, \alpha_{3}\right)$, $\alpha_{1} \geq 0, \Sigma \alpha_{1}=1$, and $T_{+}(\alpha)=\Sigma \alpha_{j} Q_{j}$. To discuss the feasible ones, we une the symbols $\alpha_{\hbar}^{(1)}$ to denote the simple allocations: $\left.\vdots\right):(1,0,0)$, etc., and let $T^{(i)}$ be the corresponding burning temperatures: $T^{(1)}=$ $T_{-}+Q_{1}$. Suppose $T^{(1)}<T^{(2)}<T^{(3)}$. Define $\bar{H}\left(T^{(j)}=H_{j}\left(T^{(j)}\right)\right.$, then $a_{i t}^{(j)}$ 
is a feasible allocation. If this is not true for any $j$, then it is a matter of checking that one of the following two possibilities must occur:

(a) there exists $T_{12} \in\left(T^{(1)}, T^{(2)}\right)$ such that $H_{1}\left(T_{12}\right)=H_{2}\left(T_{12}\right)$, or

(b) there exists $T_{23} \in\left(T^{(2)}, T^{(3)}\right)$ for which $H_{2}$ and $H_{3}$ have a common value.

In case (a), there is a feasible allocation $\left(\alpha_{1}, \alpha_{2}, 0\right)$ where $\alpha_{i}$ are chosen so that $T_{12}=\alpha_{1} T^{(1)}+\alpha_{2} T^{(2)}$. Similarly, for case (b). Hence there is always at least one feasible allocation. The same conclusion holds for any number of competing reacticas of this type.

Example 3. $\quad A_{1} \stackrel{R_{1}}{--} A_{2} \stackrel{R_{2}}{-} A_{3} \stackrel{R_{3}}{--}>P$, $V_{1}=(-1,1,0), V_{2}=(0,-1,1), V_{3}=(0,0,-1) . \quad$ Let $Y_{-}=(1,1,1)$. There is only one possibje allocation: $\alpha=(i, 2,3)$, and so the temperatures $T_{i}$ are independent of $\alpha$. We examine two of the possible cascs for their ordering. Case : : $T_{1}<T_{2}<T_{3}$. There exist suballocation $\alpha^{(1)}=(1), \alpha^{(2)}=(1,2)$, and $\alpha^{(3)}=\alpha$. The question of feasibility rests only on criterion (2). Case 2: $\mathrm{T}_{2}<\mathrm{T}_{2}<\mathrm{T}_{1}$. We reorder the reactions so that $\mathrm{R}_{3}$ becomes $\mathrm{R}_{1}$ and vice versa. With the new ordering, $\alpha=(3,2,1$,$) , nnd there are$ suballocations $\alpha^{(1)}=(1), \alpha^{(2)}=(2,1), \alpha^{(3)}=(3,2,1)$, satisiying (3.13). The following table holds:

\begin{tabular}{lllll}
$j$ & $v_{j}$ & \multicolumn{1}{c}{$v_{j}^{\prime}$} & $Q_{j}^{\prime}$ & $\hat{T}_{j}$ \\
\hline 1 & $(0,0,-1)$ & $(0,0,-1)$ & $Q_{1}$ & $T_{-}+Q_{1}$ \\
2 & $(0,-1,1)$ & $(0,-1,0)$ & $2 Q_{1}+Q_{2}$ & $T_{-}+2 Q_{1}+Q_{2}$ \\
3 & $(-1,1,0)$ & $(-1 / 3,0,0)$ & $3 Q_{1}+2 Q_{2}+Q_{3}$ & $T_{-}+3 Q_{1}+2 Q_{2}+Q_{3}$
\end{tabular}


Moreover, $\alpha^{\prime}=(1,2,3)$, so that indeed $\Sigma \alpha_{j} v_{j}=\Sigma \alpha_{j}^{\prime} v_{j}^{\prime}$. If condition (2) holds, we expect a front with reactions taking place as indicated by the $V_{j}$ : First, $A_{3} \rightarrow P$ goes to completion at $T_{1}\left(=T_{1}^{*}\right)$; then $A_{2} \rightarrow P$ goes at $T_{2}$, with $A_{3}$ an intermediate which appears only momentarily (the reaction $R_{1}: A_{2} \rightarrow P$ is much faster than $R_{2}$ at this temperature); and finally $A_{1} \rightarrow P$, pacsing through two quick intermediates.

Example 4. $A_{1} \stackrel{R_{1}}{\rightarrow} A_{2} \stackrel{R_{3}}{-}>P_{1}, A_{1} \stackrel{R_{2}}{-}>P_{2}$; $\xi=\{0\}, v_{1}=(-1,1), v_{2}=(0,-1), v_{3}(-1,0)$, and all allocations are of the form $\alpha=(\theta, 1+\theta, 1-\theta), \theta \in[0,1]$. The details are tedicus, but 1 t can be shown that allocations always exist which satisfy the feasibility criterion (1). If a feasible allocation exists with $\theta \neq 0,1$, then necessarily two of the three $T_{i}$ 's coincide. This may be the only feasible allocation in some cases, and so is imporlant. On the other hand, it would only accidentally happen that all three coincide.

4. THE LIMIT PROBLEM, LEVEL 1.

The fact is that the existence of a fearible allocation is by no ineans ensured; Example 3 above demonstrates this. If one joes exist, the formulation of the appropriate limit problem will under certain conditions, be described below. It is, in some sense a formal approximation to the front problem and has anique solution. We conjecture that there always exists an allocation satisfying criterion (1). If this allocation does not satisfy (2), then it may be possible tn find twe (or more) fronts traveling at different speeds such that the combined effect of the faster followed by the slower 1s to attain the burned state $U_{+}(\alpha)$, the various reactions 
Implied by the suballocation going to completion is the prescribed order. This is certainly true in Example 3 at the end of Sec. 3 . In general, suppose that criterion (1) holds, and also (2) for all $j$ except one, say $j=\ell$. Then it is expected that there will exist a fast front, with burning governed by the "reactions" corresponding to $\alpha^{(l)}$ and final state $\hat{u}_{\ell}=u_{-}+{ }^{k_{\ell}} a_{j}^{\prime} K_{j}^{\prime}$. There will also be a slower front with input $\hat{U}_{\ell}$ reactions described by the $v_{j}^{\prime}$ for $j>\ell$, and final state $U_{+}$.

Given a feasible allocation, we have the problem of determining the front's approximate speed and profile. The description of the procedurc will be given below for the case when all the $T_{j}$ are distinct, and $D$ is diagonal; i.e.,

$$
T_{j}=T_{j}^{*} ; D \text { is diagonal . }
$$

The point of departure is the following boundary value problem, the natural extersion of (2.1) to the case of several reactions:

$$
D U^{\prime} \cdot-\bar{M} U^{\prime}=-\sum_{j=1}^{m} w_{j}(U) k_{j}, U(-\infty)=U_{-}, U(\infty)=U_{+}(\alpha)
$$

Here the vectors $K_{j}$ are extended reaction vectors defined in Sec. 3.

The limit procedure proceeds under the approximation that all reaction rateis vanish except where $T$ is near one of the $T_{j}^{*} s_{;}$and that in the neighborhond of $T_{j}$, the requisite reactions which ensure that all $k_{k}$, for $k \leq k_{j}$, reach equilibrium, all go to completion. The principle is that the effect of those reactions at $T_{j}^{*}$ can be accounted for by prescribing a jump discontinuity in $\dot{U}$ ilt that point. 
First, we need to rescale the problem as in Sec. 2. For each $j=1, \ldots, m$, we define

$$
\begin{aligned}
& \varepsilon_{j}=T_{+}^{2} / \theta_{j}, \\
& \eta_{j}=B_{j} \varepsilon_{j}^{2} \exp \left(-\theta_{j} / T_{j}\right),
\end{aligned}
$$

and approximate

$$
w_{j}(U) \cong \frac{\eta_{i}}{\varepsilon_{j}} \psi_{j}\left(u^{(j)}\right)
$$

where $u^{(j)}=\frac{U-U_{i}}{\varepsilon_{j}}$, and $U_{j}$ is the value of $U$ (not completely knowil beforehand, except when $j=m$ ) at the temperature $T_{j}$. Here $\psi_{j}$ will be functions analogous to $(2.8)$. Let $\eta=\eta_{m}$, and rescale as (2.6). T.e result is the equation

$$
\ddot{D U}-M \dot{U}=-\sum_{j=1}^{m} \frac{r_{j}}{\varepsilon_{j}} \psi_{j}\left(\frac{U-U_{j}}{\varepsilon_{j}}\right) k_{j},
$$

where

$$
r_{j}=\eta_{j} / n
$$

If $x_{j}$ are the positions where $T=T_{j}^{*}$, the equations then become

$$
\ddot{D U}-M \dot{U}=0, x \neq x_{j}, J=1, \ldots, \text { in }^{*} \text {. }
$$




$$
\begin{aligned}
& {[D \dot{U}]_{x_{j}}=-\beta_{j} K_{j}^{\prime}, T\left(x_{j}\right)=T_{j}^{*},} \\
& U(-\infty)=U_{-}, U(\infty)=U_{+}(\alpha),
\end{aligned}
$$

where $[D \dot{U}]_{x_{j}}=\lim _{x \downarrow x_{j}} D \dot{U}(x)-\lim _{x \uparrow x_{j}} D \dot{U}(x)$, and the $K_{j}^{\prime}$ are thi. vectors defined in Sec. 3.

There are two conditions which we need to impose on the $\beta_{j}$ in order to ensure a well posed problem. According to our definition of the suballocations $\alpha^{(\ell)}$, for each of the concentration vector

$$
\hat{Y}_{\ell} \equiv Y_{-}+\sum_{j=!}^{k} \alpha_{j}^{(\ell)} V_{j}=Y_{-}+\sum_{j=1}^{\ell} \alpha_{j}^{\prime} V_{j}^{\prime}
$$

is in equilibrium with respect to the set of reactions $R_{j}, j \leq k_{\ell}$. The vector $\hat{Y}_{\ell-1}$, however, is not in this same equilibrium cone; otherwise $\alpha_{\ell}^{\prime}=0$ and we would have discarded the temperature $r_{\ell}^{*}$ and renumbered the others. This means there is some index $k(\ell)$ such that the $k-t h$ component if $\hat{Y}_{\ell}$ vanishes, but not the $K-t h$ component of $\hat{Y}_{\ell-1}$. Since $\hat{Y}_{\ell}-\hat{Y}_{\ell-1}=$ $\alpha_{l}^{\prime} V_{l}^{\prime}$, this implies that the $k-t h$ component of $V_{l}^{\prime}$ is negative, and that the coefficlent $\alpha_{\ell}^{\prime}$ is uniquely determined by the knowledge of $k($.$) . For$ notational conventence, we set $z_{k}^{0}=\left\{Y \in y_{:} Y_{k}=U\right\}$. Thus, neccessarily there is a function $K(l)$ such that $\hat{Y}_{\ell} \in \mathcal{E}_{K(l)}^{0}$, but $\hat{Y}_{\ell-1} \varepsilon_{K(l)}^{0}$. We shall require that the solution $U(x)$ of the reduced problem has the same 
property:

$Y(x) \in \varepsilon_{K(\ell)}^{0}$ for $x>x_{\ell}$.

Since $D$ is diagonal, this implies $D \dot{Y} \in \mathcal{E}_{K(\ell)}^{0}, x>x_{\ell}$. In particular,

$$
Y\left(x_{\ell}\right) \in \varepsilon_{K(l)}^{0}, D \dot{Y}\left(x_{\ell}+\right) \in \mathcal{E}_{K(l)}^{0} .
$$

Finally, there is a sa on $\beta_{m}$. First, suppose that $K_{m}=K_{m}^{\prime}$; i.e., only reaction $R_{m} 3 c$ forward at $T_{m}=T_{+}$. Then the flame layer analysis given in Sec. 2 for the decermination of $\beta$ then can also be done at $T_{r a}$; the reason essentially is that $U \equiv U_{+}=$const for $x>0$. As a result, we have

$$
\beta_{m}=\left(2 \int_{-\infty}^{0} \psi_{m}\left(s D^{-1} K_{m}\right) d s\right)^{\frac{t}{2}}
$$

On the other hand if $K_{m} \neq K_{m}^{\prime}$, then we have to account for other reactions occurring. Their rate functions will be of larger order of magnitude than that of $R_{m}$, and it turns out that a certain pseudo-steady-state approximation is valid near $T_{m}$, and reduces the analysis to the previous case. This results in a condition ( 4.11$)$ with a slightly altered $\psi_{m}$, and $K_{m}$ replaced by $K_{m}^{\prime}$.

We need to state precisely what the problem consists of.

Limit Problem, Leve1 1: Let $U_{+}, K_{j}$, and $T_{j}$ be determined from a feasible allocation $\alpha$, and assume (4.1). Solve (4.7) - (4.9) under the conditions (4.10), (4.11) (posstbly modifying (4.11) as indicated). Then the 
(approximate) actual mass flux and profile are given by

$\bar{M}=M n^{\frac{1}{2}}, \bar{U}(z)=U\left(n^{\frac{3}{2}} z\right)$.

The limit problem is easily solved. In fact, (4.7) shows that DUं $-M U$ is constant between the points $x_{j}$. In other words, $[D \dot{U}-M U]_{x_{j-1}}^{x_{j}^{-}}=0$, and (4.8) can be written [DU - MU $]_{x_{j}^{-}}^{x_{j}^{+}}=-\beta_{j} K_{j}^{\prime}$.

Summing both over $j$, from 1 to $l$, we fird

$$
[D U-M U]_{-\infty}^{x_{2}^{+}}=-\sum_{j=1}^{\ell} \beta_{j} K_{j}^{\prime}
$$

But the left side is $D U^{(}\left(x_{\ell}+\right)-M U\left(x_{\ell}\right)+M U$. We divide by M and disregard the 0 -th component of this equation, to obtain

$$
Y_{-}+\sum_{j=1}^{\ell} \frac{\beta_{j}}{M} V_{j}^{\prime}=Y\left(x_{\ell}\right)-\frac{1}{M} D Y\left(x_{\ell}+\right),
$$

which by requirement (4.10) must lie in $\mathcal{E}_{K(\varepsilon)}^{0}$. But we also know that $\hat{Y}_{\ell}=Y_{-}+\sum_{j=1}^{\ell} \alpha_{j}^{\prime} V_{j}^{\prime} \in \varepsilon_{K(\ell)}$ and $\hat{Y}_{\ell-1}$ is not. By the previous argument, the coefficients $\alpha_{j}^{\prime}$ are uniquely determined by the $K(l)$, so necessarily

$$
\frac{\beta j}{M}=a_{j}^{\prime}
$$

We determine $M$ by setting $j=m$ and using (4.11). Knowing $M$, we can find the $\beta_{j}$ for $j<m$ from (4.12) again. 
We arbitrarily set $x_{m}=0$. For $x>0, \ddot{D U}-M \dot{U}=0$, so $D \dot{U}-M U=$ const $=$ $-M U_{+}$. Thus $U=U_{+}+e^{M D^{-1}} X_{C}$ for some $C$. But since $D^{-1}$ is positive definite the only bounded solution is with $C=0$. Therefore $U(x) \equiv U_{+}$for $x>0$. From (4.8), we have $D_{\dot{u}}(0-)=\beta_{m} K_{m}^{\prime}$. Solving (4.7) now, with initial data $U=U_{+}$, DUं prescribed at $x=0$, gives

$$
U(x)=\hat{U}_{m-1}+\alpha_{m}^{\prime} K_{m}^{\prime} e^{M D^{-1} x}, x_{m-1}<x<x_{m},
$$

where

$$
\hat{U}_{m-1}=U_{+}-\alpha_{m}^{\prime} K_{m}^{\prime}=U_{-}+\sum_{j=1}^{m-1} \alpha_{j}^{\prime} K_{j}^{\prime}
$$

is the end state given by the suballocation $\alpha^{(m-1)}$. Its first component is $\hat{T}_{m-1}$, defined earlier. The function on the right of (4.i3) appraaches $\hat{U}_{m-1}$ as $x \rightarrow-\infty$, so the condition $\mathrm{r}_{m-1}>\hat{T}_{m-1}$ in the definition feasibility ensures the existence of an $x_{m-1}<0$ such that the first component of the right side of (4.13) equals $T_{m-1}$ at $x=x_{m-1}$. This determines $x_{m-1}$, as we11 as $U\left(x_{m-1}\right)$ and $D \dot{U}\left(x_{m-1}{ }^{+}\right)$. Hence $\left.\left.D \dot{U}\left(x_{m-1}\right)=D \dot{U}\right) x_{m-1}{ }^{+}\right)+\beta_{m-1} K_{m-1}^{\prime}$ is found. Integrating backwards again, we may determine successively all the $\mathbf{x}_{j}$ and the complete profile.

A comment is in order regarding assumption (4.1). As shown in Example 3 of Sec. 3, for networks with competing reactions, it may happin that a must be chosen so that two of the $T_{j}$ coincide; but it seems unlikely that there would be more than just one concident pair. If $T_{\ell}=T_{\ell+1}$, say, then the limit problem (4.7-9) looks the same exrept that for $j=\ell$, the right side of (4.8) is replaced by a linear combination of vectors $k_{k}$ for 
$k \leq k_{\ell}$, whose coefficients are not known a priori. They must be found from a flame layer analysis. The simplest example is that of the network of two competing reactions $A \rightarrow P_{1}, A \rightarrow P_{2}$. In this case, the details are given in [3].

The reason for assuming $D$ to be diagonal is to be able to cunclude (4.10) from the fact that $Y(x) \in \mathcal{E}_{(\ell)}^{0}$ for $x>x_{\ell}$. But this is only a matheratical requirement resulting from the assumption that $D$ is constant. Physically, the $K(\ell)-t h$ component of $D \dot{Y}$ represents the flux of $Y_{K(l)}$, and that flux will certainly be zero, since $Y_{K(\ell)}=0$ for $x>x_{\ell}$. Also physically, D will generally not be diagonal, but there is no contradiction because it is also nonconstant, and depends on $Y$ in such a manner that $(D \dot{Y})_{K}=0$ when $Y_{K}=\dot{Y}_{K}=0$.

\section{THE LIMIT PROBLEM, LEVEL :}

On level 1, the limit problem rontains the following apparent inconsistency: the jump conditions (4.8) replace the action of the rate functions $w_{j}(U)$ in $(4.2)$. Therefore the $\beta_{j}$ should be related to the $w_{j}$. However, the only such relation used is that for $j=m$; indeed the uther $\beta$ 's are determined uniquely as part of the solution process, with no reference to the $\omega^{\prime} s$ or $\psi^{\prime} s$. This, of course, is a great advantage in solving actual problems, because only one flasic layer analysis (namely, thai with $j=m$ ) need be performed.

It is not a true inconsistency at that level of approximation, however. To explain it, we proceed to level 2, where the relation between the $\psi^{\prime} s$ and $\beta^{\prime} s$ is brought in. 
We assume (4.1) as before, and begin by defining the "flame locations" $x_{j}$ more precisely as the positions where $Y_{K(j)}=\varepsilon_{j}$, the function $(j)$ being that in Sec. 4. We then define $U_{i} \equiv U\left(x_{j}\right)$; and in particular, $T_{j}=T\left(x_{j}\right)$. This differs from the definition of $T_{j}$ used in level 1 , where these were obtained from (3.12). The difference turns out to be small, and this justifies using the former prescription for the $T_{j}$ at the lowest level of approximation. Anticipating the small difference, we use the symbol $T_{j}^{0}$ for the sequence defined by (3.12), so

$$
H_{j}\left(r_{j}^{j}\right)=H_{m}\left(T_{+}\right) \text {for all } j,
$$

and express

$$
T_{j}=T_{j}^{0}+\varepsilon_{j} T_{j}^{\prime}
$$

Limit Problem, Level 2. Let the $U_{+}, K_{j}^{\prime}$, and $T_{j}^{0}$ be detcrmined f:om a feasible allocation $\alpha$, and assume (4.1). Solve (4.7) - (4.9) for $M, U(x)$, $x_{j}$, and $\beta_{j}$ under conditions $(4.10),(4.11)$. Also delermine the $T_{j}^{\prime}$ for $j<m$ in accordance with the flame layer analyses to be described below. Then $\bar{M}$ and $\bar{U}(z)$ are found as before.

In the case $K_{j}=K_{j}$, the flame layer analysis procecds ty using stretched variables

$$
\xi=\frac{x-x_{j}}{\varepsilon_{j}}, u^{(j)}=\frac{u-U_{j}}{\varepsilon_{j}}
$$


the flame equation analogous to $(2.9)$ is then

$$
\frac{\partial^{2} u^{(j)}}{\partial \xi^{2}}+r_{j} \psi_{j}\left(u^{(j)}\right) k_{j}=0
$$

It turns out that for any given vectors $P_{+}$with $0-t h$ component $\geq 0$ and $p_{-}=p_{+}+\beta_{j} K_{j}$, there exists a positive number $r_{j}$ such that (5.2) has a solution satisfying

$$
D \frac{\partial u(j)}{\partial \xi}( \pm \infty)=p_{ \pm} .
$$

This problem of delermining $r_{j}$ can be reduced to a standard problem of Liñan [5], [4]. The $p_{ \pm}$have already been determined from (4.7) - (4.9) as before; this now gives us $r_{j}$. Finally, taking the 108 of (4.6), using $(4.4)$ and $(3.10)$, we find

$$
\text { E. } \ln r_{j}=\left(H_{j}\left(T_{j}^{0}+c_{j} T_{j}^{\prime}\right)+2 c \ln \varepsilon_{j}\right)-\left(H_{m}\left(T_{+}\right)+2 \varepsilon \ln \varepsilon_{m}\right) \text {. }
$$

We write

$$
\left.H_{j}\left(T_{j}^{0}+\varepsilon_{j} T_{j}^{\prime}\right) \cong H_{j}\left(T_{j}^{0}\right)+\varepsilon_{j} H_{j}\left(T_{j}^{0}\right) T_{j}^{\prime}=H_{j}\left(T_{j}^{(0)}\right)+\underset{T_{j}}{\left(T_{+}^{0}\right.}\right)^{2} \varepsilon_{j} T_{j}^{\prime} .
$$

Hence from (5.1),

$$
\ln r_{j}=\underset{T_{j}}{\left(-T_{+}\right)^{2}} T_{j}+2 \ln \left(\ddot{c}_{i_{m}}^{c_{j}}\right) .
$$


This determines $T_{j}^{\prime}$. If $\frac{\varepsilon_{j}}{\varepsilon_{m}} \neq 0(1)$, then $\left|T_{j}^{\prime}\right|>0(1)$, but at least the temperature $\operatorname{corr}:$ tions $\varepsilon T_{j}^{\prime} \ll 1$, since $\varepsilon=\underset{i}{\min \varepsilon_{i}}$.

In the sase $K_{i}^{\prime} \neq K_{j}$, the comments in Sec. 4 regarding pseudo-steadystate ni:1.j: pply here us well.

6. FU!?RL UIRECTIONS

Many aspects of the present treatment remain to be fully explored, for example, procedures for finding all possible feasible allocations, a systematic flame layer analysis in cases when $K_{j} \neq K_{j}^{\prime}$, more than formal justification, itc. More importantly, the theory needs to be extended to cases ([6] being an example] where some reactions have zero or amall activation energy. This is all in the context of plane laminar flames. But other crucial problems in flame theory abound; the significance of multiple reactions is them needs to be explored. Some of these directions are under current active investigation.

\section{BIBLIOGRAPIIY}

1. II. Bereutyckf, B. Ntcolannko, hnd B. Scheurer, "Traveling Wave Solutions to ?eaction Diffuston Syatems Modeling Comburtion," to appear in Yroceedirigy of Special Summer Confernce oti Partial Differental Equations, Durham, NH, June 1982; American Mathematjcal Society.

2. J. Buckmanter and G. S. S. ludford, "Theory of louminar rlames," Conbridge. Unjueratey Preas, 1982.

3. P. C. Hife and H. Nicolaenko, "The ningular perturbalion appronch to flame theory with chain and competing reactions," to appear in Proceeding Conference on Differential Fifuntions, Dumiec, (1982), Bpringer-Verjag leceture Noten in Mathemation. 
4. P. C. Fife and B. Nicolaenko, "Asymptotic flame theory wit." complex chemistry," in preparation.

5. H. Hastings and $A$. Poore, "On a nonlinear differential equation from combustion theory," to appear.

6. A. Liñan, "A theoretical analysis of premixed flame propagation with an isothermal chain reaction. Instituto Nacional de Tecnica Aerospacial "Eateban Terradas" (Madrid), USAFOSR Contract No. EOOAR 68-0031, Technical Report No. 1.

PAUL C. FIFE

DEPARTMENT OF MATHEMATICS

UNIVERSITY OF ARIZONA

TUCSON, AZ 87521

BASIL NI COI.AENKO

CENTER FOR NONLINEAR STUDIES, MS B258

LOS ALAMOS NATIONAL LABORATORY

LOS ALAMOS, NM 87545 